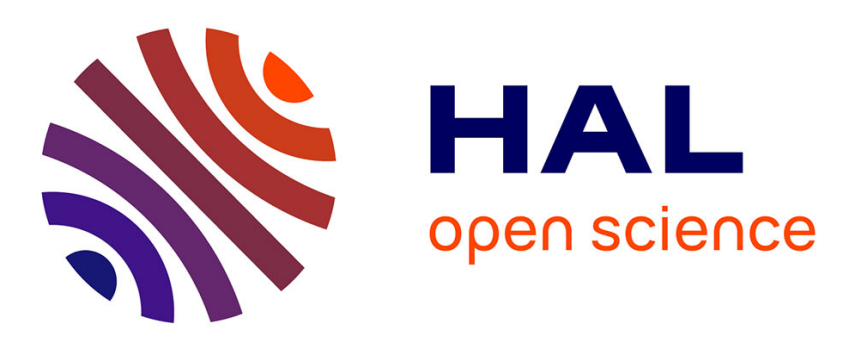

\title{
Micromechanics of Coalescence: Synergism Between Elasticity, Plastic Yielding and Multi-Size Scale Voids
}

\author{
J. Faleskog, C. Fong Shih
}

\section{To cite this version:}

J. Faleskog, C. Fong Shih. Micromechanics of Coalescence: Synergism Between Elasticity, Plastic Yielding and Multi-Size Scale Voids. Journal de Physique IV Proceedings, 1996, 06 (C6), pp.C6-91C6-100. 10.1051/jp4:1996609 . jpa-00254437

\section{HAL Id: jpa-00254437 https://hal.science/jpa-00254437}

Submitted on 1 Jan 1996

HAL is a multi-disciplinary open access archive for the deposit and dissemination of scientific research documents, whether they are published or not. The documents may come from teaching and research institutions in France or abroad, or from public or private research centers.
L'archive ouverte pluridisciplinaire $\mathbf{H A L}$, est destinée au dépôt et à la diffusion de documents scientifiques de niveau recherche, publiés ou non, émanant des établissements d'enseignement et de recherche français ou étrangers, des laboratoires publics ou privés. 


\title{
Micromechanics of Coalescence: Synergism Between Elasticity, Plastic Yielding and Multi-Size Scale Voids
}

\author{
J. Faleskog and C. Fong Shih \\ Division of Engineering, Brown University, Providence, RI 02912, U.S.A.
}

\begin{abstract}
Void growth and coalescence under physical states similar to those found in highly stressed regions ahead of a crack is investigated. The analysis introduces a representative material volume containing several large voids and a population of microvoids present from the very beginning, all of which are modeled as discrete entities. Plastic yielding has pervaded the material volume of interest. The underlying micromechanics of final rupture is dominated by a succession of rapidly growing microvoids. This involves the synergistic interaction between elasticity associated with high stress triaxiality, stiffness softening caused by plastic yielding and a rich supply of length scales arising from voids of vastly different sizes.
\end{abstract}

\section{INTRODUCTION}

Ductile fracture in many metal alloys begins with the nucleation of cavities from the brittle cracking or decohesion of inclusions. As these cavities grow in size, they generate locally intense fields around neighboring small inclusions thereby nucleating small-scale cavities which participate in the final phase of the coalescence process. This tearing process comprising distinct stages of nucleation, growth and coalescence of cavities has been observed in numerous experimental investigations (see review articlessby Van Stone et al. [1], and Garrison and Moody, [2]). The process of cavity growth is fairly well understood (McClintock, [3]; Rice and Tracey, [4]) and model's for the growth phase are quite advanced (see review article by Needleman, Tvergaard and Hutchinson, [5]). However the mechanisms of nucleation and coalescence and the associated micromechanics are less well understood. It is clear that improved understanding of the above mechanisms and their effect on failure modes and fracture resistance can facilitate the development of mechanism-based fracture models for implementation in predictive tools for nonlinear fracture mechanics analysis (e.g. Rousselier, [6]; Bilby, Howard and Li, [7]; Xia, Shih and Hutchinson, [8]).

In flat dimple fracture in steel (example, see figure 5.3, p. 306, in Anderdson [9]) the holejoining process occurs over a macroscopically planar zone of thickness comparable to the spacing between large voids. The fracture surface is in this case completely filled with voids of vastly different size scales. These voids originate from the various inclusions. A rich source of inclusions notwithstanding, many of the larger cavities do not display evidence of having originated from large sulphide or oxide inclusions. This is consistent with rough estimates indicating that there are not enough sulphide or oxide inclusions to account for the numerous large cavities found at a fracture surface (Zhang and Link, [10]). It appears that some of the larger voids might have originated from carbides.

A distinctly different hole-joining mode can be found in some high strength alloys. As an example see figure 22, in [1]. In this mode of coalescence, large inclusions are linked by bands of localized plastic shearing. Microcavities which are one to two orders of magnitude smaller are found within these bands. Here the coalescence process occurs by the joining of small-scale cavities leading to what is referred to as void-sheet formation (Rogers, [11]) between sulphide-nucleated voids. In this study, we will attempt to elucidate the underlying micromechanics relevant to the coalescence processes which dominate the ductile failure modes described above. 


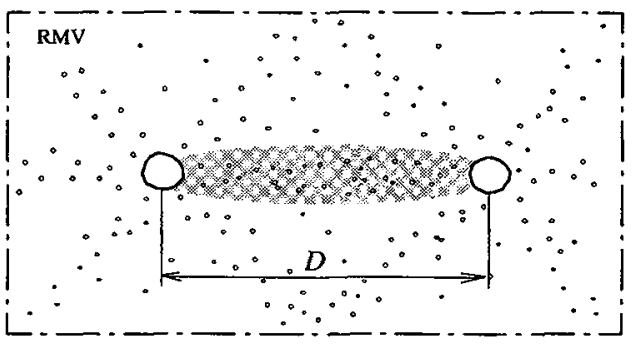

Figure 1: A representative material volume, RMV, containg two large voids and a distribution of microvoids. In a high constraint case microvoids located in the shaded zone are those most likely to participate in the coalescence process.

A typical metal alloy has several size scales of inclusions with different mean spacings which provide a rich spectrum of size scales for fracture. In experiments on copper, Palmer, Smith and Warda [12] found void nucleation sites at $\mathrm{SiO}_{2}$ particles of diameter as small as $5 \mathrm{~nm}$. In steel, voids can nucleate from various inhomogeneities e.g. sulphide and carbide inclusions. Carbide particle diameters range between tens and hundreds of nanometers. Large particles like sulphides and oxides have diameters ranging from one to ten microns and the mean spacing between large particles is on the order of a hundred microns [10]. Suppose $\ell_{s}$ and $\ell_{l}$ denote the mean sizes of small and large particles and $D$ represents the mean spacing between large particles. Then the ratio $\ell_{l} / D$ falls between $10^{-1}$ and $10^{-2}$, while $\ell_{s} / D$ is considerably smaller, between $10^{-3}$ and $10^{-4}$.

Now consider a representative material volume (RMV) situated in the peak stress region ahead of the crack front. This material volume contains two large voids and a large population of small inclusions as illustrated in Fig. 1. It is assumed that a fraction of small inclusions is weakly bonded so that these inhomogeneities can be treated as pre-existing microvoids. The RMV is subjected to a macroscopic field of high hydrostatic stress. The main features of the coalescence process are now described. Under increasing stress the large void grows in concert with the plastic strain. Simultaneously, a local zone of high stress concentration emanates from the large void and spreads across the material raising the stresses at nearby microvoids. As a result, the hydrostatic stress surrounding one or more microvoids is raised to a level that activates an unstable deformation mode in which the stored elastic energy drives the plastic expansion of the microvoid. Although the overall stress decreases rapidly, small zones of high stress concentration are generated near growing voids. These zones engulf smaller nearbylmicrovoids which now grow rapidly. This process continues until the submicron ligament fails by microcleavage or by shearing along crystallographic planes.

Under typical circumstances, the RMV containing the microvoids has already been extensively yielded. The critical stress level for rapid plastic expansion of the microvoid is related to the tangent stiffness of the plastically strained material. Since the latter is significantly lower than the elastic modulus, prior plastic straining has the effect of lowering the critical stress level for rapid microvoid growth. It will be shown that material nonlinearity in the form of decreasing stiffness with increasing plastic strain can lower the critical stress for rapid void growth to levels that are found in the peak stress region ahead of a crack front. That is, plastic yielding plays a crucial role in the coalescence process driven by microvoid cavitation.

A physically-based approach for ductile rupture by hole growth mechanism is adopted in this study. A pre-existing void of size $\ell / D=10^{-4}$ can be regarded as a minute benign void. This nascent microvoid exerts no effect on the macroscopic field with characteristic length on the order of $D$ or larger. By contrast, large voids can elevate the fields locally around nearby microvoids. We describe the coalescence process in which the underlying micromechanics is found in the synergistic interaction between elasticity associated with high stress triaxiality, stiffiness softening caused by plastic yielding and a rich source of size scales arising from voids of vastly different sizes. Cavitation is viewed as an elasticity-driven phenomenon whereby a minute, benign void undergoes rapid, dramatic growth reaching a size set by the characteristic length of the locally elevated stress field. 
In some cases, this microvoid can attain a size comparable to the larger neighboring voids. In other cases, the cavitation phenomenon is shorter-lived. Here the enlarged microvoids remain significantly smaller than neighboring large voids. In formulating the mechanics framework for our physical viewpoint, we will make contact with the related approaches to cavitation instabilities in elastic-plastic solids taken by Huang, Hutchinson and Tvergaard [13] and Hou and Abeyaratne [14]. Investigations into such instabilities began with Bishop, Hill and Mott [15] and Hill [16]. Ball [17] studied this phenomenon as an intrinsic material instability within the theory of nonlinear elasticity. Readers are referred to the above papers for different treatments of the phenomenon. For a more complete treatment of the current problem see Faleskog and Shih [18].

\section{MICROMECHANICS MODEL}

\subsection{Cell volume and some preliminaries}

A typical cell volume contains one centrally positioned large void and a number of microvoids. All voids are modeled as discrete cylindrical cavities. Only a small number of microvoids could be treated because the regions surrounding the microvoids must be modeled by highly refined meshes. Therefore it is important to place microvoids in regions of elevated stress and/or intense plastic deformation. In most cases, microvoids are equidistantly placed on the horizontal plane and on the diagonal plane emanating from the large void. In this study, all voids are present at the very beginning of straining; in effect the voids have nucleated at zero stress and strain. The cell volume can be regarded as one of many in a periodic microstructure with large voids arranged in a square pattern with distance to nearest neighbor initially equal to $D$. All voids, including microvoids, are modelled as cylindrical holes and plane strain conditions are assumed. On a macroscale, the material is subjected to a biaxial strain state $E_{1}$ and $E_{2}\left(E_{3}=0\right)$. These are the logarithmic strains and the work conjugate quantities are the true (Cauchy) stresses $\Sigma_{1}, \Sigma_{2}$ and $\Sigma_{3}$. The quantity $\frac{1}{3}\left(\Sigma_{1}+\Sigma_{2}+\Sigma_{3}\right)$ defines the macroscopic hydrostatic stress $\Sigma_{h}$.

The cell is subjected to proportional straining such that the imposed logarithmic strains satisfy

$$
\psi_{\epsilon}=\frac{E_{1}}{E_{2}}
$$

at all times. Under plane strain conditions $\left(E_{3}=0\right)$, the remote strain state is fully specified by $\psi_{\epsilon}$ and one additional parameter, e.g. $E_{2}$. The biaxiality of the remote strain can serve as an indicator of constraint. Values of $\psi_{\epsilon}$ in the range -0.95 to 1.0 are considered in our analysis.

The displacement of a material particle labeled $\mathbf{X}$ in the initial configuration and $\mathbf{x}$ in the deformed configuration is $\mathbf{u}=\mathbf{x}-\mathbf{X}$. Periodic displacement boundary conditions are applied on the quarter geometry of the cell volume to be analyzed $-0 \leq X_{1} \leq D / 2,0 \leq X_{2} \leq D / 2-$ of the form :

$$
\begin{array}{llll}
u_{1}=0 & \text { at } & & X_{1}=0 \\
u_{2}=0 & \text { at } & & X_{2}=0 \\
u_{1}=U_{1} & \text { at } & & X_{1}=D / 2 \\
u_{2}=U_{2} & \text { at } & & X_{2}=D / 2
\end{array}
$$

The relations between $U_{1}, U_{2}$ and the logarithmic strains are

$$
E_{1}=\ln \left(1+\frac{2 U_{1}}{D}\right), \quad E_{2}=\ln \left(1+\frac{2 U_{2}}{D}\right)
$$

In addition all boundaries are shear traction free and all void surfaces are entirely free of tractions. The above conditions are applicable to all calculations under discussion.

For the full range of strain biaxialities, $-1 \leq \psi_{\varepsilon} \leq 1$, the corresponding range of stress biaxialities is $0 \leq \psi_{\sigma} \leq 1$, where

$$
\psi_{\sigma}=\frac{\Sigma_{1}}{\Sigma_{2}}
$$


The range of strain biaxialities of interest to the present work falls within -0.7 and 0 . This corresponds to the strain biaxialities reported for a growing crack (Xia and Shih, [19]).

The strain biaxialities for a blunted stationary crack tip in the vicinity of maximum hoop stress are slightly lower.

For the specific range of strain biaxialities, $-0.8 \leq \psi_{\varepsilon} \leq 0$, the cell analysis to be discussed in Section 3 resulted in computed stress ratios, $\psi_{\sigma}$, within the range from 0.6 to 0.8 . These in-plane stress ratios are in agreement with those associated with the HRR singularity and those found from full field analyses for stationary cracks (see O'Dowd and Shih, [20], for details). The stress ratios ahead of growing cracks can be slightly higher as can be inferred from the computational studies by Varias and Shih [21] and [19].

\subsection{Finite element procedure and constitutive relations}

The finite element calculations employ the fully implicit integration scheme developed by Cuitiño and Ortiz [22] for a finite strain $J_{2}$ plasticity theory based on multiplicative kinematics. In this framework stresses are related to strains through a hyperelastic potential defined on an intermediate configuration. A linear elastic response is assumed on this configuration and the elastic material constants are given by Young's modulus, $E$ and Poisson's number, $\nu$. In all the calculations $\nu$ was equal to 0.3 . form

In the present work, power law strain rate sensitivity and strain hardening are assumed in the

$$
\dot{\bar{\varepsilon}}^{\mathrm{p}}= \begin{cases}\dot{\varepsilon}_{0}\left[\left(\frac{\sigma_{\mathrm{e}}}{H\left(\bar{\varepsilon}^{\mathrm{p}}\right)}\right)^{1 / m}-1\right] & \text { if } \sigma_{\mathrm{e}} \geq H\left(\bar{\varepsilon}^{\mathrm{p}}\right) \\ 0 & \text { if } \sigma_{\mathrm{e}} \leq H\left(\bar{\varepsilon}^{\mathrm{p}}\right)\end{cases}
$$

with

$$
H\left(\bar{\varepsilon}^{\mathrm{p}}\right)=\sigma_{0}\left(1+\frac{\bar{\varepsilon}^{\mathrm{p}}}{\varepsilon_{0}}\right)^{N}
$$

In the above equations, $\bar{\varepsilon}^{\mathrm{P}}$ is the equivalent plastic strain, $\dot{\varepsilon}_{0}$ a reference strain rate, $m$ the strain rate sensitivity exponent and $\sigma_{\mathrm{e}}$ the effective (Mises) stress. $H$ is an isotropic hardening function of $\bar{\varepsilon}$ with exponent $N, \sigma_{0}$ the initial yield stress, and $\varepsilon_{0}$ the reference strain $\left(\varepsilon_{0}=\sigma_{0} / E\right)$.

The present study is restricted to rate-independent material behavior obtained from the above equations for a sufficiently small strain rate exponent. In the present case $m=0.005$. In all computations, the finite element model employs six-noded triangles with quadratic interpolation for the displacement field. Full integration based on three interior points was used.

\section{SINGLE VOID RESULTS-GENERAL FEATURES}

The mechanics of the growth of a single void can provide new insights into the synergistic interplay between elasticity, plasticity induced reduction of tangent stiffness, and voids with vastly different size scales. by

At this point, it is convenient to introduce the initial and current void volume fractions defined

$$
f_{0}=\frac{v_{0}}{V_{0}}, \quad f=\frac{v}{V}
$$

where $v$ and $V$ refer to the void and cell volumes, respectively, and the subscript 0 designates the initial value. For a cylindrical void, $f_{0}=(\pi / 4) \times(\ell / D)^{2}$.

We introduce the subject under discussion by displaying three sets of results for the macroscopic stress versus the macroscopic strain of a unit cell computed under three different strain biaxialities, see Fig. 2(a). Proportional remote straining throughout the entire loading is imposed by a fixed value of $\psi_{\epsilon}$ and monotonically increasing the strain $E_{2}$. The material parameters are $\sigma_{0} / E=0.002$ and $N=0.1$. We now direct our attention to the set of traction-strain curves labelled by $\psi_{\epsilon}=0.0$. For $f_{0}=10^{-3}$, the stress peaks at about $4.2 \sigma_{0}$. Beyond this point, the stress decreases because 

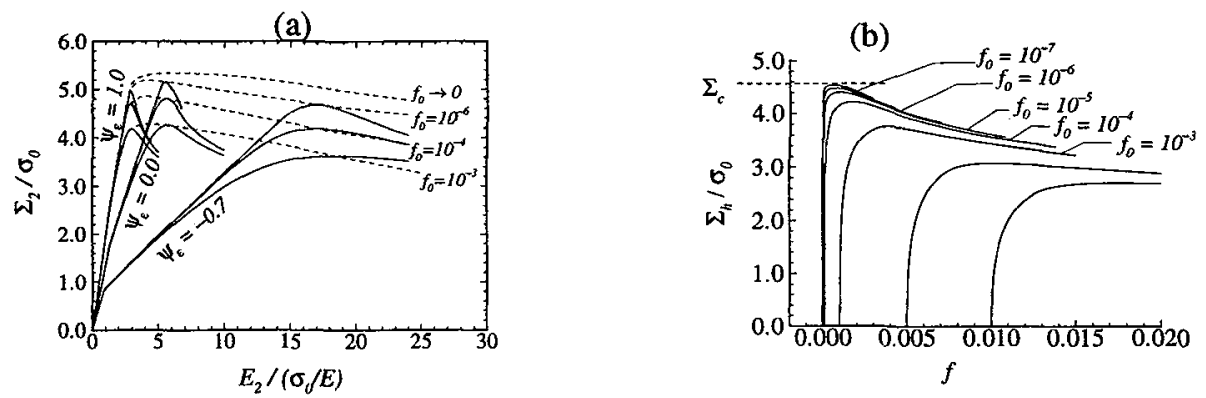

Figure 2: (a) Traction-strain curves of a cell volume (single void) showing the influence of macroscopic strain biaxialities and decreasing $f_{0}$ values. (b) Hydrostatic stress as a function of the evolution of void volume fraction $(f=v / V)$ in a cell volume (single void) under uniaxial straining, $\left(\psi_{\epsilon}=0\right)$. The maximum hydrostatic stress corresponding to the limiting case $f \rightarrow 0$. is marked by $\Sigma_{c}$.

material strain hardening is unable to compensate for the strain softening due to the increase of hole size through rapid void growth. For smaller values of initial cavity size, $f_{0}$ equal to $10^{-4}$ and $10^{-6}$, the stresses peak at higher values but they fall off very rapidly in the post-peak stress regime. This rapid growth phenomenon becomes very pronounced as $f_{0}$ decreases.

In all cases under discussion, both the peak stress and the maximum void growth rate, $d v / v d E_{2}$, are preceded by the onset of overall plastic yielding. The trends displayed in Fig. 2(a) show the reduction in the peak stress with increasing overall plastic yielding ( $\psi_{\varepsilon}$ becoming negative). These results provide strong evidence that material nonlinearity plays a crucial role in setting the stress level for rapid void growth.

Our calculations based on imposed strain boundary conditions show that the void growth rate, $d v / v d E_{2}$, reaches its maximum just before the stress peak is reached. We will use the term critical stress to designate the stress at maximum void growth rate. As the initial void size becomes smaller and smaller, the critical stress approaches the peak stress from below. As a matter of fact, the stresses at maximum void growth rate for $f_{0}=10^{-8}$ are practically identical to the peak stresses.

The peak stresses for a fixed value of $f_{0}$ form an envelope over the range of biaxial strain ratios considered $\left(\psi_{\varepsilon}:-0.80\right.$ to 1.0$)$. Envelopes for $f_{0}$ equal to $10^{-3}, 10^{-4}$ and $10^{-6}$ are indicated in Fig. 2(a) by the dashed lines.

Now consider the limiting situation $f_{0} \rightarrow 0$ corresponding to an infinitesimal void $v \rightarrow 0$ within a finite body of initial volume $V_{0}$. At a fixed value of $\psi_{\epsilon}$, the peak stress as $f_{0} \rightarrow 0$ can be obtained by extrapolation using a sequence of solutions for decreasing $f_{0}$ 's This limiting stress as a function of $\psi_{\epsilon}$, over the range -0.80 to 1.0 , is shown by the dashed line in Fig. 2(a). labeled by $f_{0} \rightarrow 0$. This represents the cavitation stress envelope for rapid expansion of a pre-existing infinitesimal void driven by the elastic energy stored in the finite body. This event could be interpreted as a cavitation instability as investigated by [13]and [14].

The full behavior of $\Sigma_{h} / \sigma_{0}$ versus $f$ is plotted in Fig. 2(b) for seven values of $f_{0}$ ranging from $10^{-2}$ to $10^{-7}$. The strain biaxiality is $\psi_{\epsilon}=0$. It can be seen that a higher level of hydrostatic stress is attained as $f_{0}$ is decreased. At elevated stress levels, the drop in the stresses in the postpeak regime is more pronounced indicative of rapid void expansion. The cavitation stress, $\Sigma_{c}$, is indicated by the dashed line. Our sequence of solutions for $f_{0} \rightarrow 0$ shows that the cavitation stress is approached rapidly and is effectively attained once $f_{0}$ is less than about $10^{-8}$.

\section{CAVITATION CRITERION}

The effect of prior plastic yielding on the stress level for cavitation can be inferred from the trend of the cavitation stress envelope $\left(f_{0} \rightarrow 0\right)$ in Fig. 2(a). This plastic strain softening effect can be rationalized by the decreasing material tangent stiffness due to increasing plastic strain. To 

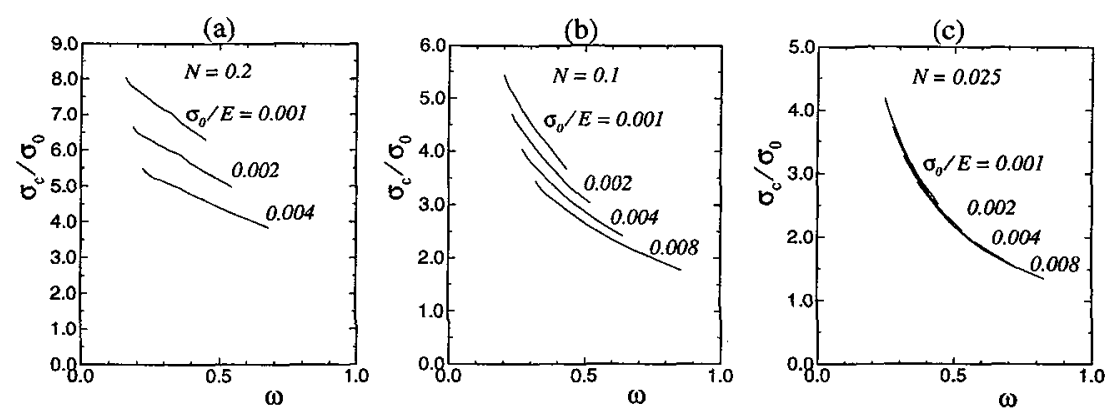

Figure 3: The dependence of hydrostatic stress at cavitation on the parameter $\omega$ for several values of $\sigma_{0} / E$ : (a) $N=0.2$; (b) $N=0.1$; (c) $N=0.025$.

account for this, we introduce a parameter $\omega$ defined by $\sigma_{e} / \sigma_{h}$. The characteristic length of the field under discussion is large compared to the size of the microvoid so that the above stresses can be interpreted as local point-wise quantities. Under continuous plastic deformation and within $J_{2}$ flow theory of plasticity, a one-to-one relationship exists between $\sigma_{e}$ and the single physical state variable, the equivalent plastic strain. It must follow that the current tangent stiffness is a function of $\omega$. A large value of $\omega$ represents a higher level of plastic deformation which should bring about a reduction in the cavitation stress. A similar observation was made by [14] for a spherical void subjected to axisymmetric tension.

An operational definition for the cavitation stress is suggested by the physical viewpoint described in the Introduction and the results presented in Section 3. Guided by our observations, we phrase the criterion for cavitation under biaxial straining (plane strain) in terms of the attainment of a critical hydrostatic stress, $\sigma_{c}$. This stress is defined by the value of $\sigma_{h}$ corresponding to the maximum growth rate, $\left(\dot{v} / v \dot{E}_{2}\right)_{\max }$, of a void with $f_{0}=10^{-8}$. The cavitation stress depends on material parameters, $\sigma_{0} / E, N, \nu$ and the physical state characterized by $\omega$.

The results pertinent to the present discussion are shown in Figure 3. These are computed for strain biaxialities over the range -0.95 to 1.0. The hydrostatic stress levels at cavitation are displayed in the form of curves of $\sigma_{c}$ versus $\omega$. Results are presented for three values of the strain hardening exponent and several values of $\sigma_{0} / E$. It can be seen that low hardening and high yield stress can significantly lower the stress for cavitation, $\sigma_{c} / \sigma_{0}$.

Building upon the above observations and the physical picture described in the Introduction, we adopt a local cavitation criterion based on the hydrostatic stress:

$$
\begin{array}{ll}
\mathcal{C}<0 & \text { cavitation not possible } \\
\mathcal{C} \geq 0 & \text { cavitation possible }
\end{array}
$$

where

$$
\mathcal{C}\left(\sigma_{\mathrm{h}}, \sigma_{\mathrm{c}}\right)=\sigma_{\mathrm{h}}-\sigma_{\mathrm{c}}\left(\omega ; \sigma_{\mathrm{o}} / E, N, \nu\right) .
$$

Here $\sigma_{\mathrm{h}}$ is the local hydrostatic stress and $\sigma_{c}$ is the cavitation stress discussed above. This criterion should be interpreted in the following way. If the criterion is met at a material element containing a minute microvoid, it will be possible for this void to undergo extremely rapid expansion; this rapid expansion will continue until the void starts to interact with its surrounding field.

\section{MULTIPLE SIZE SCALE VOIDS AND THE LINK-UP PROCESS}

\subsection{Diverse zones fulfilling cavitation criterion}

In the Introduction it was alluded that the interplay between material parameters and constraint can give rise to distinctly different coalescence modes. We will now attempt to explain this interplay by way of the two different coalescence modes discussed in the introduction. 


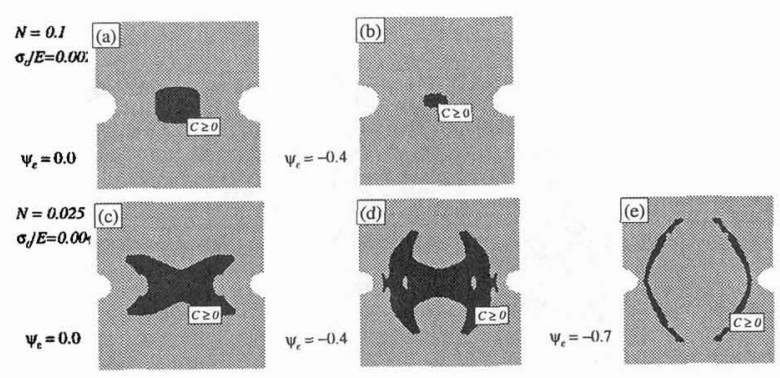

Figure 4: Contour maps of $\mathcal{C}$ for two different materials, $(\mathrm{a}, \mathrm{b}) \sigma_{0} / E=0.002, N=0.1$ and $(\mathrm{c}, \mathrm{d}, \mathrm{e}) \sigma_{0} / E=0.004$, $N=0.025$ and several strain biaxialities as indicated in the figures.

(a)

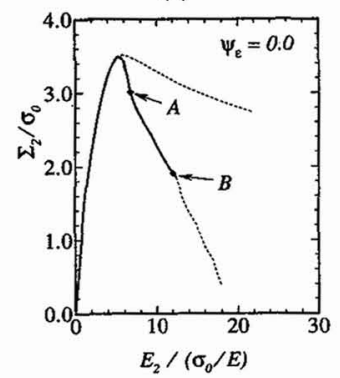

(b)

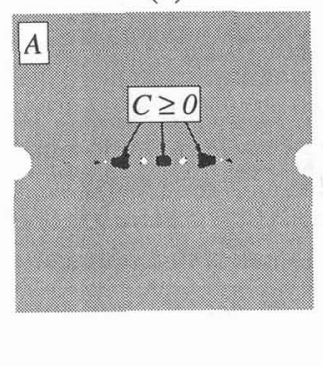

(c)

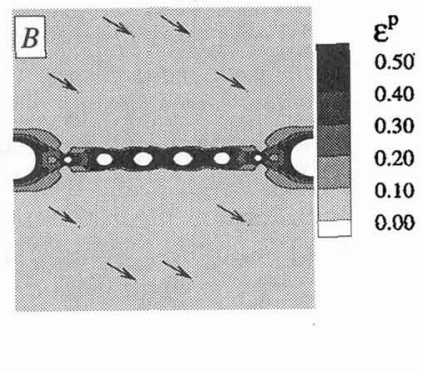

Figure 5: Flat dimple rupture mode of coalescence under high strain biaxiality in a medium strength alloy $\left(\sigma_{0} / E=\right.$ $0.002, N=0.1$ ). (a) Macroscopic stress-strain behavior featuring cascading softening. (b) Cavitated microvoids and current cavitation zones at load point $A$. (c) Advanced coalescence state at load point $B$, displaying contours of effective plastic strain.

We begin the discussion on flat dimpled rupture mode of coalescence by studying contour maps of zones in which the cavitation criterion (9) is fulfilled. These zones were identified by applying the cavitation criterion introduced in Section 4 to the solution of a single void in a representative material volume discussed in Section 3. The zone indicated in black in Fig. 4(a) is the region satisfying the criterion, $C \geq 0$, under high strain biaxiality, $\psi_{\varepsilon}=0$. For lower strain biaxiality, the cavitation zone size is significantly reduced as can be seen in Fig. 4(b). These zones represent the maximum extent of the region in which the cavitation criterion is met under the present loading history.

The cavitation zones displayed in Fig. $4(\mathrm{a}, \mathrm{b})$ were obtained for a medium strength metal alloy with moderate amount of hardening $\left(\sigma_{0} / E=0.002, N=0.1\right)$. In this analysis the initial size of representative large holes relative to their spacing is $0.08 D\left(=\ell_{l} / D\right)$, corresponding to $f_{0}=0.005$.

A corresponding set of results for a high strength metal alloy with low hardening is given in Fig. $4(\mathrm{c}, \mathrm{d}, \mathrm{e})\left(\sigma_{0} / E=0.004, N=0.025\right)$. At high strain biaxiality, Fig. 4(c), the cavitation criterion is met in the black zone and this is similar to the one shown in Fig. 4(a). At lower strain biaxialities, the size and shape of the cavitation zones are drastically different, see Fig. $4(\mathrm{~d})$ and (e). The latter indicate that microvoids aligned within a narrow band could be expected to grow rapidly to form a void-sheet mode of coalescence linking the large voids.

\subsection{Two modes of coalescence assisted by microvoid cavitation}

To test the veracity of our cavitation criterion, we augment the single void cell model introduced in Section 3 by including a small population of microvoids. These microvoids are modelled as 
(a)

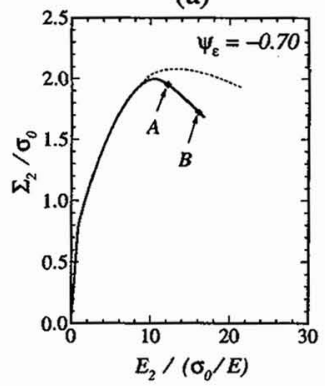

(b)

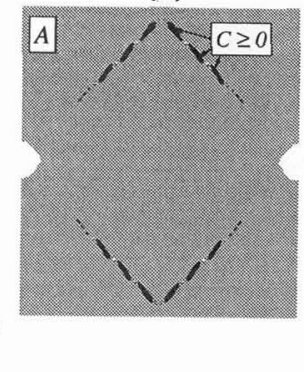

(c)

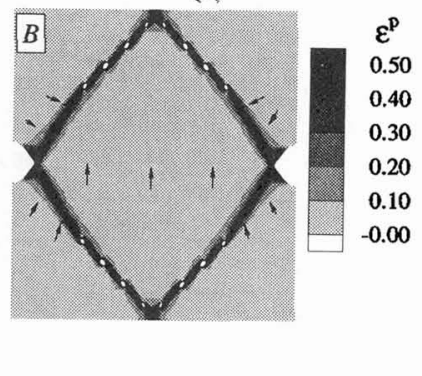

Figure 6: Void-sheet mode of coalescence under low strain biaxiality in a high strength alloy $\left(\sigma_{0} / E=0.004, N=0.025\right)$. (a) Macroscopic stress-strain behavior. (b) Cavitated microvoids and current cavitation zones at load point $A$. (c) Advanced coalescence state at load point $B$, displaying contours of effective plastic strain.

discrete cylindrical holes and placed in locations where the cavitation criterion could be met, e.g. along the horizontal axis and along the diagonal of the cell. The size of these microvoids are $\ell_{s} / D=0.001$ and microvoids of this size were used in all calculations reported in this section. The size of the large void in each cell correspond to $f_{0}=0.005$ and is the same as in Section 5.1.

We begin by discussing the flat dimpled rupture case. Fig. 5(a) displays the stress-strain behavior of the cell under high strain biaxiality, $\psi_{\varepsilon}=0.0$. The response up to the peak stress is unexceptional. When microvoids are absent, the load in the post-peak stress regime drops gradually as shown by the dashed line. However, as can be seen the presence of microvoids results in a dramatic drop in the load. The accelerated load drop to point ' $A$ ' is the result of microvoid cavitation shown in Fig. 5(b). Here, the largest microvoids have enlarged their volumes by a factor of 630 . At the same time, the growth rate of the large void has been substantially decreased because of the reductions in the macroscopic stress. The zones currently satisfying the cavitation criterion are indicated in the figure. These zones are determined by the local fields generated by the newly enlarged microvoids and the existing large voids. Fig. 5(c) displays a late state in the coalescence process. Here, it is evident that the plastic strain has localized within a narrow band on the order of the void size. The largest microvoids have by now enlarged their volumes by a factor of 2900 . The macroscopic stress at this advanced coalescence state is indicated by ' $B$ ' in Fig. 5(a). Observe that microvoids away from regions of intense cavitation exhibit insignificant growth as indicated by black dots marked by arrows.

Within the computational resources available to this study, we could incorporate only a small number of microvoids into the computational model. Needless to say, a typical ductile rupture process in a metal alloy involves many more microvoids and size scales of inclusions. Now, suppose that additional microvoids of similar or even smaller size scales are embedded in the highly strained material indicated by darker shades in Fig. 5(c). When these minute microvoids are engulfed by locally elevated stresses they will in turn cavitate repeating the events described above. This pattern of cavitation and void growth gives rise to cascading load drops (indicated by dashes) in Fig. 5(a) until finally the submicron ligament fails by microcleavage or by shearing along crystallographic planes.

We now turn our attention to coalescence by void-sheet formation between sulphide-nucleated voids. Figure $6(\mathrm{a})$ shows the stress-strain behavior of a cell which contains one large void, $f_{0}=$ 0.005 , subjected to low strain biaxiality $\left(\psi_{\varepsilon}=-0.7\right)$. Here, the relative peak stress is considerably lower than for the previous case. The macroscopic stress in the post-peak stress regime falls off smoothly when microvoids are absent. When microvoids are present, the stress drops more rapidly. The enlarged microvoids at stress levels indicated by ' $A$ ' and ' $B$ ' are shown in Fig. 6(b) and (c). It can be seen that the growth rate of the large voids has been greatly reduced while the microvoids aligned along the diagonal continue to enlarge driven by evolving high local stresses. Such evolution 
of high local stresses can be inferred from the map of the current cavitation zones indicated in Fig. 6(b). At an advanced state, the intense plastic shearing is confined to a narrow band width on the order of the size of the enlarged microvoids. This band, joining large sulphide-nucleated voids, is populated by cavitated microvoids. The microvoids in Fig. 6(b) and (c) have enlarged their volumes by 140 and 440, respectively. It is worth noting that microvoids outside this band remain in their nascent, benign state as indicated by the black dots marked by arrows.

\section{DISCUSSION}

The highly coupled nature of the processes leading to final coalescence as depicted in Figs. 5 and 6 appears to be central to explaining both coalescence modes discussed in the introduction. It also sheds light on a long-standing question concerning the role of cavity formation on localization of plastic flow. On this question, two different sequences of events have been put forth: nucleation and growth of small cavities is the precursor to the formation of plastic localization, and alternatively plastic flow first localizes creating the conditions for the nucleation of small cavities and their growth (Rice [23], see page 208). Our work suggests that the two processes are coupled, one driving the other, thereby escalating the chain of events to final coalescence.

To understand the nature of the coupling under discussion, we review the results presented in Figs. 5 and 6 . Here the high triaxiality macroscopic field prevails over a distance greater than the microstructural length $D$. Driven by this field, the large void enlarges and emits a local zone of high stress concentration. The microvoids embedded in the nearby softened material and subjected to this elevated local field are stressed to cavitation-like conditions. These microvoids experience rapid growth and simultaneously generate intense local plastic deformation as well as high stresses. Now, the above process repeats itself with the next size scale of microvoids. The above scenario suggests that microvoid growth and formation of a localized band of high plastic strain is a synergistic process in which the growth of microvoids drives the intensification of the strain which in turn causes more microvoids to cavitate and grow. The essential role of multiple length scales in this process is evident.

The simplest model for flat dimpled rupture assumes that final fracture occurs by localized necking of the remaining ligament between the large void and the current crack tip (Rice and Johnson, [24]). Motivated by this model, finite element analyses have been carried out by Aravas and McMeeking [25]for cylindrical voids and by Hom and McMeeking [26] for spherical voids under plane strain conditions. They studied the interaction between the discrete voids and the crack front under increasing load attempting to make a quantitative connection between fracture toughness and microstructural parameters, e.g. spacing between large particles, $D$, and the particle diameter $\ell$. The role of shear localization in the void link-up process was considered by Tvergaard [27]. In his $2-D$ finite element analyis the large voids were modeled as discrete entities and the smaller voids were modeled by the Gurson constitutive relation (Gurson, [28]). Tvergaard found that the void growth process could be terminated abruptly by plastic shear localization between the enlarged voids resulting in rapid void link-up. Building upon this observation, Needleman and Tvergaard [29] studied crack growth in a material containing two size scales of voids. They observed that plastic shear localizations between enlarged voids and the crack tip set the limits on the achievable fracture toughness.

The present work was undertaken to gain some insight into the role of primary and secondary inclusions on the coalescence process. In the initial phase of a study of this nature, it is convenient to work with a tractable model based on cylindrical voids. Obviously, the real microstructures and inclusions are three-dimensional in nature. An initial study within an axisymmetric cell model using two discrete voids of slightly different sizes has been carried out by Tvergaard [30]. We do expect that a full three-dimensional treatment along the lines of the present work using sphere-like voids will confirm the characteristic features elucidated upon in this study. This will allow a more direct evaluation of the present approach with behavior arising from realistic microstructures. Such study is in progress. 


\section{Acknowledgements}

This investigation is supported by Grant N61533-93-K-0030 from David Taylor Research and Development Center funded by the Nuclear Regulatory Commission and by Grant N00014-95-1-0399 funded by the Office of Naval Research. In addition JF was supported by grants from Swedish Research Council for Engineering Sciences and from The Foundation BLANCEFLOR BoncompagniLudovisi, née Bildt, Stockholm, Sweden.

\section{References}

[1] Van Stone, R. H., Cox, T. B. Low, J. R. Jr. and Psioda, J. A., International Metals Reviews 30 (1985) 157-179.

[2] Garrison, W. M. Jr. and Moody, N. R., The J. Phys. Chem. Solids 48 (1987) 1035-1074.

[3] McClintock, F. A., J. Appl. Mech. 35 (1968) 363-371.

[4] Rice, J. R. and Tracey, D. M., J. Mech. Phys. Solids 17 (1969) 201-217.

[5] Needleman, A., Tvergaard, V. and Hutchinson, J.W., "Void Growth in Plastic Solids", Topics in Fracture and Fatigue, A. S. Argon Ed. (Springer Verlag, New York, 1992) pp. 145-178.

[6] Rousselier, G., Nucl. Engng Design 105 (1987) 97-111.

[7] Bilby, B.A., Howard, I.C. and Li, Z.H., Fatigue Fract. Engng Mater. Struct. 16 (1993) 1-20.

[8] Xia, L., Shih, C.F. and Hutchinson, J.W., J. Mech. Phys. Solids, 43 (1995) 389-413.

[9] Anderson, T. L., Fracture Mechanics-Fundamentals and Application, 1st ed. (CRC Press, Boca Raton, Florida, 1991) p. 306

[10] Zhang, X.J. and Link, R.E. (1995) Private communication.

[11] Rogers, H. C., Trans. Metall. Soc. AIME 218 (1960) 498-506.

[12] Palmer, I. G., Smith, G. C. and Warda, R. D., Physical basis of yield and fracture, A. C. Strickland Ed. (The Physical Society, London, 1967) p. 53.

[13] Huang, Y., Hutchinson, J.W. and Tvergaard, V., J. Mech. Phys. Solids 39 (1991) 223-241.

[14] Hou, H.-S. and Abeyaratne, R., J. Mech. Phys. Solids 40 (1992) 571-592.

[15] Bishop, R. F., Hill, R. and Mott, N. F., Proc. Phys. Soc. 57 (1945) 147-159.

[16] Hill, R., The Mathematical Theory of Plasticity (Clarendon Press, Oxford, 1950) pp. 97-127.

[17] Ball, J. M., Phil. Trans. R. Soc. London A306 (1982) 557-611.

[18] Faleskog J.P. and Shih, C.F., J. Mech. Phys. Solids, accepted for publication 1996.

[19] Xia, L. and Shih, C.F. J. Mech. Phys. Solids 43 (1995) 1953-1981.

[20] O'Dowd, N. P. and Shih, C.F., J. Mech. Phys. Solids 39 (1991) 989-1015.

[21] Varias, A. G. and Shih, C. F., J. Mech. Phys. Solids 41 (1993) 835-861.

[22] Cuitiño, A. and Ortiz, M., Engng Computations 9 (1992) 437-451.

[23] Rice, J. R., "The localization of plastic deformation", 14th International Congress on Theoretical and Applied Mechanics, W. T. Koiter Ed. (Delft, North-Holland, 1976) Vol. 1, pp. $207-220$.

[24] Rice, J. R and Johnson, M. A., "The role of large crack tip geometry changes in plane strain fracture", Inelastic Behaviour of Solids, M. F. Kanninen, W. F. Adler, A. R. Rosenfield and R. I. Jaffe, Eds. (McGraw-Hill Series in Material Science and Engineering, New York, 1970) pp. 641-672.

[25] Aravas, N. and McMeeking, R. M. J. Mech. Phys. Solids 33 (1985) 25-49.

[26] Hom, C. L. and McMeeking R. M. J. Mech. Phys. Solids 37 (1989) 395-415.

[27] Tvergaard, V. J. Mech. Phys. Solids 30 (1982) 265-286.

[28] Gurson, A. L. J. Engng Mater. Technol. 99 (1977) 2-15.

[29] Needleman, A. and Tvergaard, V, J. Mech. Phys. Solids 35 (1987) 151-183.

[30] Tvergaard, V. (1995) Effect of void size difference on growth and cavitation instabilities. Submitted for publication. 